\title{
A case report: simple papillary muscle vegetation caused by infective endocarditis and confirmed by PET-CT
}

\author{
qing xia ${ }^{1}$, Yao $\mathrm{Qu}^{1}$, and Qianqian Sheng $^{1}$
}

${ }^{1}$ Affiliation not available

November 7, 2020

\begin{abstract}
Infective endocarditis was a cause that can not be ignored in patients with clinical fever, which was often missed and misdiagnosed because of the diversity of clinical manifestations. This paper reported a case of infective endocarditis diagnosed by echocardiography and PET-CT due to recurrent fever. The patient was an elderly woman with a history of 20 years of type 2 diabetes and 5 years of hypertension. The first symptom was recurrent fever. After admission, the relevant examination did not determine the cause, and later echocardiography found that the papillary muscle vegetation was attached and diagnosed as infective endocarditis.
\end{abstract}

\section{Hosted file}

Body text.pdf available at https://authorea.com/users/373834/articles/491447-a-case-reportsimple-papillary-muscle-vegetation-caused-by-infective-endocarditis-and-confirmed-bypet-ct 